PÁGINA ABERTA

\title{
La trascendencia del sumak kawsay para nuestra comprensión de la educación, la ética y la política
}

Mario Madroñero Morillo a Enma Polonia Campozano Aviles ${ }^{b}$ Julio Uyaguari ${ }^{c}$

\section{Resumen}

Sumak kawsay es una concepción que en el transcurso de los últimos años ha generado considerable interés en contextos políticos, económicos, educativos, de investigación, recibiendo atención por parte de activistas y académicos debido a la "ruptura" que provoca sobre la concepción actual de orden mundial. En diferentes contextos, se asume como algo que debe ser alcanzado a través de un proceso de transformación sobre la manera en la que se construyen las representaciones sociales a nivel singular, comunitario y planetario al proponer "pensar de otra manera" los conceptos de la modernidad que damos por sentados, y al promover un llamado a la restauración o invención del sentido de la experiencia, la cultura, la vida, de tal manera que pueda existir armonía y respeto con la naturaleza.

Palabras clave: Suma kawsay. Ética. Política. Educación. Transformación.

\section{Introducción}

Sustentado en el pensamiento indígena andino, pero en continua apertura, el sumak kawsay cuenta con numerosas interpretaciones que lo relacionan con una ruptura con el orden establecido. Para Acosta (2008) "son muchas las voces que se han sumado a esta noción", pues tal como evoca un líder Quechua, la atención al concepto se debe a que "no es una propuesta indígena solo para los pueblos indígenas, sino que concierne a toda la sociedad" (MACAS, 2010, p. 452; GUDYNAS, 2011), debido a que propone la construcción social de subjetividades a través de vías alternas para el establecimiento de relaciones diferentes con el

\footnotetext{
a Universidad Nacional de Educación, Cañar, Ecuador.

b Universidad Nacional de Educación, Cañar, Ecuador.

c Universidad Nacional de Educación, Cañar, Ecuador.
} 
mundo (CORTEZ, 2011), que implican alternativas éticas (GUDYNAS, 2011) relevantes para la concepción sobre la formación por la trascendencia de su sentido.

En esta perspectiva, el primer aspecto a tratar se dedica a la reflexión sobre lo que implica el sumak kawsay como un concepto en permanente construcción y sobre su presencia en contextos políticos, que lo proponen como base para sustentar una idea de educación relacionada con el buen vivir. En este sentido, exploramos el concepto a partir de su contexto sociolingüístico, estableciendo relaciones entre los diferentes elementos y componentes que lo sustentan como una posibilidad de poner en práctica otros modos de formación desde la construcción de políticas educativas diferentes.

Comprender qué es el sumak kawsay conlleva interpretar qué implica su sentido para la construcción de procesos de formación, considerando que el concepto es polilógico por las dos palabras que lo componen y el contexto en el que se propone. La primera es sumak que significa plenitud/esplendor (MACAS, 2010), la segunda es kawsay que significa ser-siendo, la mezcla de los significados y sentidos en el concepto componen la interpretación del sumak kawsay como "vivir la vida en el presente", en interrelación e interacción con los otros sentidos de la existencia; a partir de esta comprensión, sumak kawsay representa la vida como magnificencia, provocada por una relación inmanente y trascendente entre los seres y la naturaleza.

Para comprender cuáles serían sus implicaciones éticas/educativas para la concepción de formación, identificamos como bases teóricas los siguientes elementos: relacionalidad, ética, política y conocimiento expuestos en la Figura 1, que permiten tratar el segundo aspecto referido a su operacionalización, es decir a cómo toma lugar en documentos sobre política - asumiendo de acuerdo con Gudynas (2011) -, que es en el nivel político en donde se corre el riesgo de neutralizar el sumak kawsay al clausurar su sentido al interpretarlo como un estado de plenitud en el que no habría rupturas (GUDYNAS, 2011). A partir de este punto, nos enfocaremos en cómo la educación es afectada al considerar sus conceptos y propuestas.

En este contexto, estudiamos la concepción sobre el sumak kawsay y los conceptos y prácticas que subyacen a la misma, a partir de su comprensión como Buen Vivir y Vida Plena con dos propósitos: el primero, comprender la visión de comunidad implícita en esta filosofía; y el segundo, distinguir la trascendencia que el sumak kawsay presenta a nuestra concepción de "educación", desde una perspectiva política, ética y formativa. 
Figura 1 - Elementos

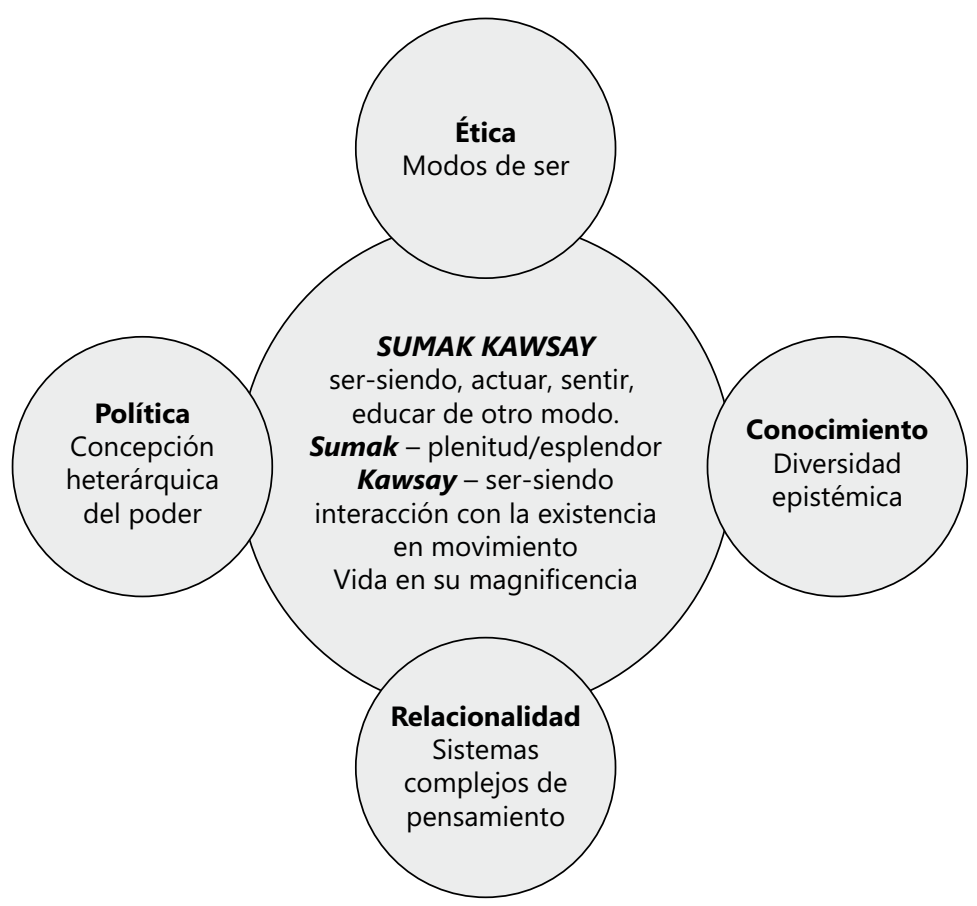

Fuente: Elaboración propia (2018)

\section{La concepción teórica sobre el sumak kawsay y su relación con la educación}

La definición propuesta por Macas (2010) describe el sumak kawsay como un "sistema" cuya racionalidad corresponde a las naciones Abya-Yala, sustentada en una serie de principios éticos que confluyen en el "estar siendo" (kawsay), que en nuestra perspectiva componen un ecosistema de transformación ontológica que relacionado con la educación hace posible proponer una política educativa transformativa, concebida para incentivar el "estar siendo" como formación de un ser abierto a la transformación singular y comunitaria.

El ecosistema de tal política educativa se trama entre los siguientes conceptos: "Yachay" en tanto conocimientos abiertos a la comunidad, "Ruray"/"Maki-maki", comprendido como el hacer mano a mano el trabajo comunitario, "Ushay" como el poder de organización política y comunitaria, "Munay" en tanto deseo y donación, que componen una dimensión ontológica y ética que se propondría en el 
concepto "Kay" comprendido como el ser-siendo, correspondiente a los siguientes principios o "condiciones": "Rantiranti" concebido como reciprocidad; "Puray" entendido como relacionalidad; "Yanantin" asumido como complementariedad y "Tinkuy" propuesto como encuentro entre los componentes de una realidad que se concibe inacabada e inconstante, que refleja una política transformativa en diáspora, en un continuo de emancipación en diseminación por su inacabamiento y contemporaneidad como se expresa en las Figuras 2 y 3.

En el pensamiento amerindio el ser se concibe como incompleto, pensamiento que se expresa en la palabra kay que hace mención a un ser inconstante y en devenir continuo, singularidad que permite proponer que se trata de una ontología transformativa que provoca una ética y una política de la re-moción del ser que generan un proceso de transformación y conllevan la praxis del des-aprendizaje de modos de ser que no correspondan a un buen vivir o a una vida plena; característica que sustenta una política educativa transformativa que en el contexto de la formación tendría consecuencias en las siguientes dimensiones.

\section{Figura 2 - Conceptos}

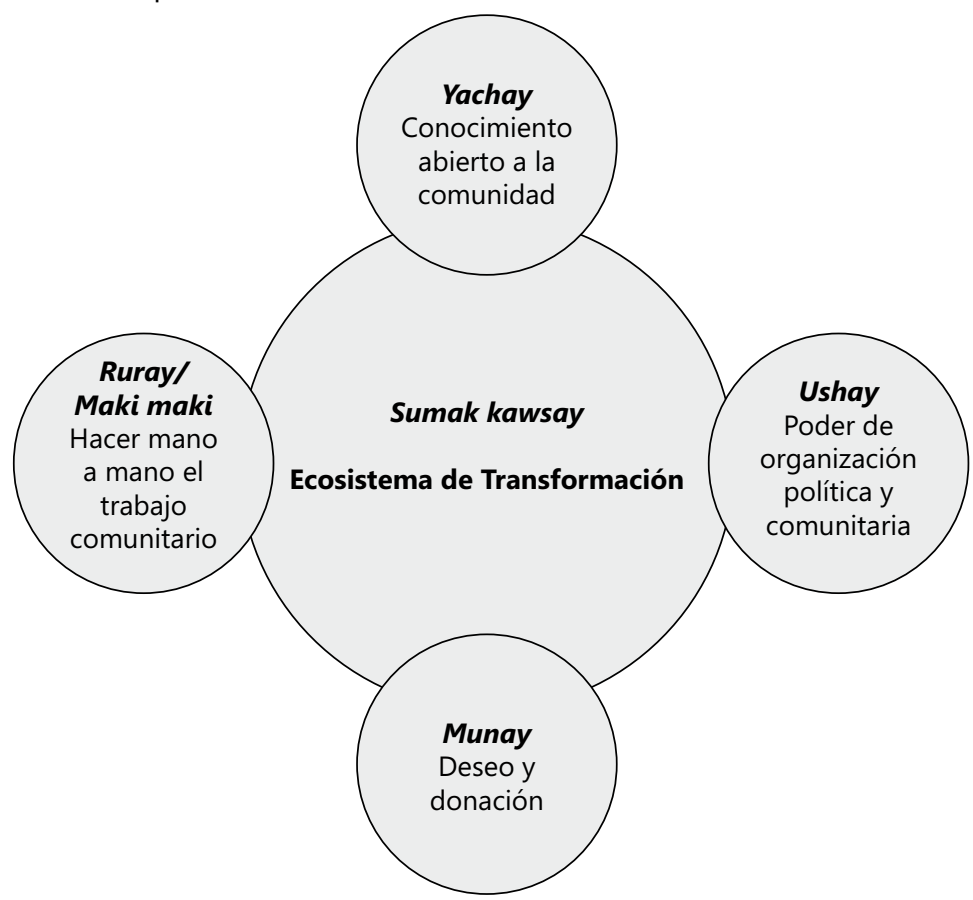

Fuente: Elaboración propia (2018) 
Figura 3 - Condiciones

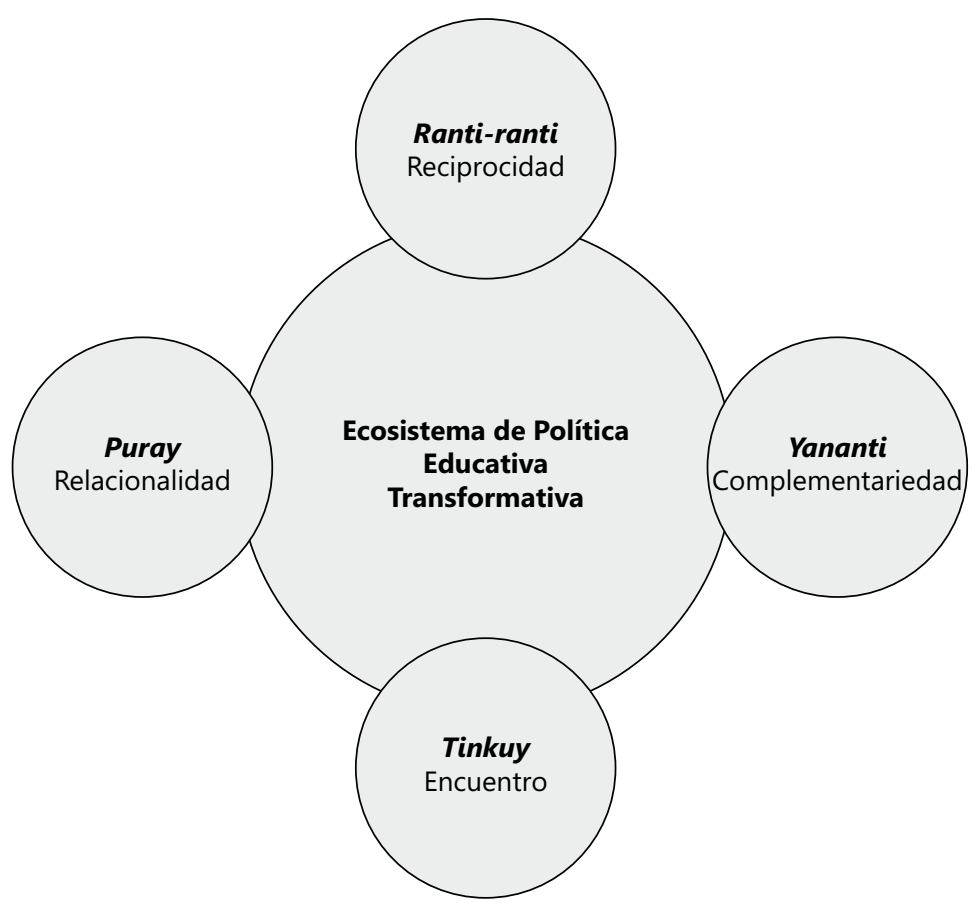

Fuente: Elaboración propia (2018)

a) ontológicas, en relación con el proceso de transformación del ser del docente y el estudiante,

b) éticas, en correspondencia a los modos de existencia del docente y estudiante investigadores y la generación de valores que provoquen,

c) poéticas, en cuanto a la comprensión del tiempo de la formación y la creación de conceptos y saberes que promuevan emancipación,

d) estéticas, en la medida en la que se trata de la praxis de un pensamiento creativo y de transformación desde la innovación, re-moción o creación de modos de existencia alternativos,

e) científicas y epistemológicas, en cuanto a la dimensión dialógica entre diversidades epistémicas, conceptos inter y transculturales sobre praxis científicas y tecnologías, 
f) políticas y económicas, en referencia a las decisiones de aprendizaje y reciprocidad dirigidas a la producción de saberes y conocimientos en correspondencia con la diversidad epistémica,

g) pedagógicas, en cuanto a las diferentes prácticas, estrategias y tácticas inovadoras de una educación transformativa ${ }^{1}$.

Las dimensiones serían parte del ecosistema de la política educativa transformativa de una posible práctica de formación desde el sumak kawsay, que conlleva la proposición de una re-moción del ser en el docente y el estudiante, que plantea establecer una relación con el conocimiento desde la diversidad epistémica en reciprocidad, con la intención de provocar una economía de las relaciones de poder en relación con el saber, referidas al disenso como expresión del reconocimiento de la diferencia, comprendida como la forma de manifestación de la inconstancia del ser abierto a la transformación como se representa en el Figura 4.

\section{Rastreando el sumak kawsay desde su formulación teórica hasta su incorporación en documentos sobre política}

La política educativa transformativa que genera el sumak kawsay provoca revoluciones que conllevan repensar la realidad de la política contemporánea; en este sentido, las transformaciones sociales y las crisis que las representan se asumen como síntomas de la decadencia de un sistema político que, a pesar de estar en colapso, continúa funcionando de manera automática.

El automatismo político y la estructura dialéctica que lo constituye genera sistemas de autoinmunidad que le permiten sostenerse y validarse como fundamento único para la comprensión de la realidad global; de esta forma garantiza su conservación e inserción en procesos que buscan provocar cambios, regulando inclusive el devenir singular, comunal o minoritario de los movimientos sociales o de otras expresiones de revolución que pudieran interrumpir la lógica de la permanencia que lo caracteriza.

La lógica disruptiva del sumak kawsay confronta la dialéctica de "la deuda, el deber y la obediencia" (AGAMBEN, 2008) que producen la autoinmunidad y la inercia del automatismo político, al provocar una disrupción en el sistema a partir de la diferencia ontológica y la alter-natividad (TROUILLOUT-ROLPH,

\footnotetext{
Una propuesta con variaciones se propone en: Ruano; Morillo; Gonzáles (2018). Esta versión la precede y se presentó en formato de ponencia en la European Conference on Educational Research. University College Copenhagen, Denmark, 21 to 25 august, 2017.
} 
Figura 4 - Dimensiones

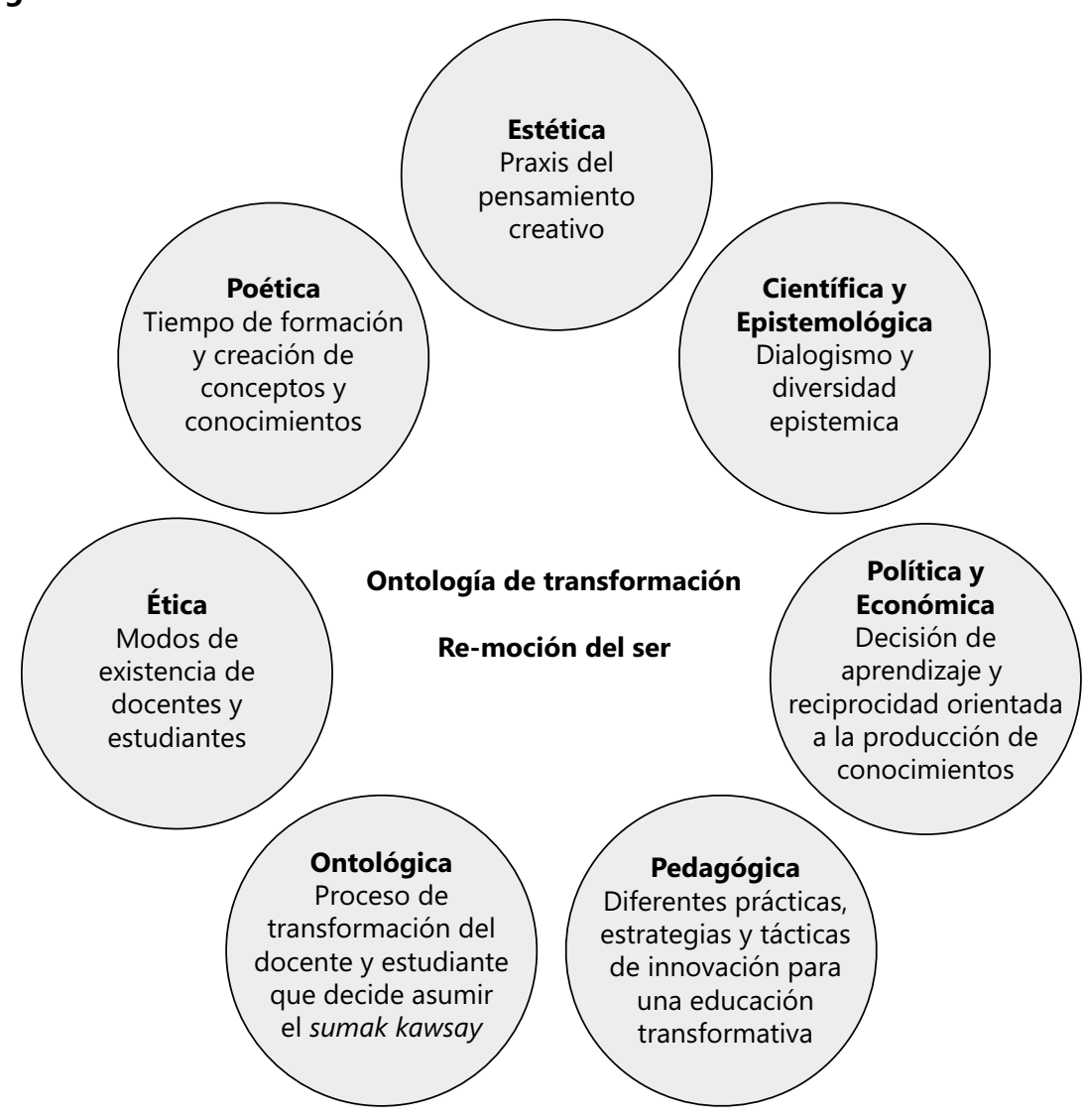

Fuente: Elaboración propia (2018)

2011), que afectan las relaciones consigo mismo, la comunalidad (RIVERA CUSICANQUI, 2017)2 ${ }^{2}$, la naturaleza, el mundo, el cosmos, el universo.

La diferencia ontológica y la alter-natividad se asumen como prácticas políticas de reafirmación de la vida con el lugar o ecosistema que la revela y sustenta, generando un vínculo distinto con la tierra que provoca una geopolítica de las relaciones que motiva la proposición de una cosmopolítica, asumida como la comprensión de la complejidad de las relaciones entre los poderes del tejido social de una realidad plural, como destaca Grosfoguel (2016):

\footnotetext{
Texto inédito, presentado en el XVI congreso de antropología: Políticas de los conocimientos y las prácticas antropológicas de América Latina y el Caribe, Universidad Javeriana, Bogotá, Colombia, 6 al 9 junio de 2017.
} 
[...] en las cosmovisiones "no-occidentales" de las epistemologías del sur (Sousa Santos, 2010) - que no participan de la visión dualista del mundo, sino que tienen dentro de sí la noción holística de diversidad dentro de la unicidad (por ejemplo, la "Pachamama" en los indígenas andinos, "Twaheed" en el Islam, "Ubundu" en África, etc.) - hay una visión completamente distinta. En la visión holística no existe "naturaleza", sino el "cosmos" y todos estamos al interior del mismo como formas de vida interdependientes que co-existen entre sí. Esto produce la racionalidad de que la vida humana no está fuera del sistema ecológico y es dependiente de las otras formas de vida. La vida humana es concebida al interior de la ecología planetaria y, por tanto, si destruyes tu eco-sistema o las otras formas de vida a tu alrededor te destruyes a ti mismo. De manera que la ecología y sus diversas formas de vida y existencia no son un medio hacia otro fin sino un fin en sí mismo. Cualquier tecnología que se construye a partir de ese principio lleva dentro de sí la racionalidad de la reproducción de la vida (GROSFOGUEL, 2016, p. 129).

El cuidado de la vida (STIEGLER, 2010) expone un biocentrismo que hace parte de esta perspectiva cosmopolítica que hace posible comprender las relaciones que se establecen en un territorio, asumido como un ecosistema de correspondencias entre poderes diferentes, que sustenta por ejemplo, las nociones de "Estado pluri-nacional", derechos de la naturaleza (Pacha mama) y el "sumak kawsay" (LAJO, 2011), asumidos como objetivos de gobierno en Ecuador y en Bolivia (con algunas diferencias), al proponer un sistema de comprensión de la política desde la diversidad epistémica.

La atención que las constituciones de Ecuador y Bolivia dan a estas concepciones presenta una perspectiva sobre la política que se distancia de la noción de república clásica y de la noción moderna que la prolonga en las estructuras jerárquicas que la constituyen. La diferencia con los sistemas políticos de organización de una comunidad radica en que se proponen principios políticos desde una perspectiva heterárquica (KONTOPOULOS, 1993), en la que comparecen poderes dispares que no pretenden establecer relaciones de homogeneidad, sino de disenso, tal como expone el concepto aymara Chi'xi (RIVERA-CUSICANQUI, 2010) que:

[...] conjuga el mundo indio con su opuesto, sin mezclarse nunca con él. [...] plantea la coexistencia en paralelo de múltiples diferencias culturales que no se funden, sino que antagonizan o se complementan. Cada una se reproduce a sí misma desde 
la profundidad del pasado y se relaciona con las otras de forma contenciosa (RIVERA-CUSICANQUI, 2010, p. 70).

El antagonismo como complementariedad no se limita a una dialéctica de contrarios en este ecosistema de relaciones, pues provoca paradojas críticas que remueven los fundamentos de las concepciones de ley, derecho, deber, justicia y libertad, haciendo del sumak kawsay un principio deontológico que provoca formas alter-nativas para pensar la justicia, relacionadas con la exposición de "nuevos sujetos de derecho", a través de la relación crítica entre "una globalización y descolonización" del derecho internacional (LIU-SHING, 2009, p. 26) que provoca repensar los fundamentos sobre los que se han estructurado las nociones de soberanía, Estado, nación, comunidad, sujeto, género. En el caso del contexto político del Ecuador, repensar los fundamentos ha permitido la concepción de los derechos de la naturaleza en donde la tierra se asume como sujeto de derecho, pues el concepto Pacha Mama no es personificado a través de un procedimiento metafórico que lo sublime en una alegoría, sino que lo concibe como una alteridad.

La Pacha Mama representa para el pensamiento amerindio la alteridad de la tierra que en tanto ecosistema de pensamiento alter-nativas, sustenta la generación y gesta de políticas transformativas a través de los diferentes "procesos de alteración diferencial" (VIVEIROS DE CASTRO, 2002) de las diversas prácticas de conocimiento, relacionadas con los siguientes aspectos, presentes en la Figura 5:

a) Concepción heterárquica de la noción de poder y creación;

b) comprensión multinatural (VIVEIROS DE CASTRO, 2002) del territorio, la región, la localidad, el cuerpo;

c) perspectivismo y diversidad epistémica en relación a la comprensión de la percepción de la realidad y la construcción de los conocimientos;

d) una ética política de la donación como práctica de la reciprocidad desde el disenso;

e) un ecosistema político educativo transformativo.

La incorporación de estos aspectos en un sistema político recalca la necesidad de una ciencia política más atenta a los procesos de formación de comunalidad (RIVERA-CUSICANQUI, 2017), que incentivan la incorporación de concepciones como las del sumak kawsay en contextos políticos como los de Ecuador y Bolivia, 
Figura 5 - Aspectos

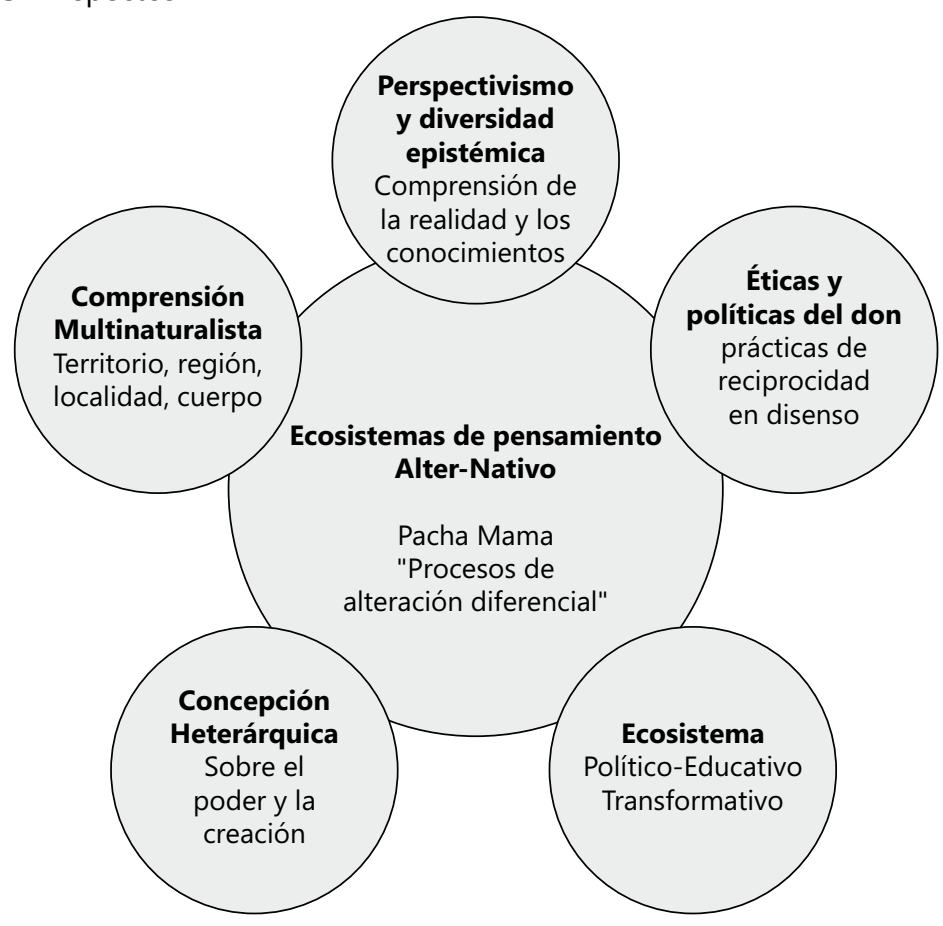

Fuente: Elaboración propia (2018)

que han asumido el riesgo de provocar paradojas políticas que ponen en entredicho las formas en las que se han concebido los Estados desde la instauración de la globalización en tanto régimen de representación económico y político restringido.

El proceso de incorporación de conceptos del pensamiento amerindio como el sumak kawsay genera crisis individuales y sociales inevitables, pues se trata de sistemas de pensamiento complejo que rebasan la noción moderna de cosmovisión y conllevan la emergencia de cosmografías que sustentan una concepción geopolítica ecosistémica plural que descentraliza la razón al dislocarla de los nichos conceptuales en los que se fundamenta, desmantelando la estructura de la "fuerza de ley" y el "fundamento místico de la autoridad" (DERRIDA, 2008) por la que se han impuesto.

El desmantelamiento corresponde a una crítica del principio de soberanía, por lo que las nociones de Estado, república, nación, se ponen en entredicho para proponer frente a su insuficiencia la noción de Estado plurinacional; concepción que se 
sustenta en una paradoja de representación, pues la noción de plurinacionalidad se propone como un contrapunteo epistémico para soportar la gravedad de la concepción de Estado.

Lo plurinacional es en este sentido una inversión categórica del principio de autoinmunidad y conservación del Estado, relacionado con una concepción heterárquica (KONTOPOULOS, 1993) del poder, el saber, la autoridad y por tanto de la producción de la verdad y la gubernamentalidad. La inversión categórica de la autoinmunidad es una consecuencia de la diáspora de la razón que provoca la diversidad epistémica que permite interpretar el poder, el saber, la autoridad, desde la emergencia de nociones de verdad y gobierno diferentes, que no solo son críticas, pues se caracterizan por la creación e invención de conceptos.

La dimensión creativa o heurística que provoca la relación con un pensamiento diferente es parte del ecosistema de la política educativa transformativa relacionada con el sumak kawsay, que en Ecuador ha provocado la propuesta de una reinvención del Estado - con las consecuencias que esto conlleva a las formas en las que están constituidas sus estructuras jurídicas, legislativas y judiciales -, por lo que la reforma constitucional se promueve como un proceso irreversible que se asume desde la radicalización por la conservación de lo establecido, hasta la exacerbación de un deseo de transformación de la realidad, que hace de estas formas de lo político manifestaciones de lo que se podría comprender como una política del exceso que intenta regular la reciprocidad en disenso y la heterarquía que genera.

\section{Consideraciones sobre su validez conceptual y capacidad operativa, aplicada en sistemas educativos}

La aplicación de concepciones como el sumak kawsay provoca crisis al interior de los sistemas de representación que constituyen una realidad. En el contexto educativo no es diferente, en la medida en la que se ponen en entredicho las dimensiones ontológicas, éticas, políticas, estéticas, epistémicas, económicas, jurídicas, pedagógicas, que conforman la estructura académica de un sistema educativo y las maneras en las que concibe la formación para la construcción de realidad al regular las dimensiones mencionadas por medio de áreas y asignaturas, a través de las cuales la crisis se extiende a docentes y estudiantes.

La crisis corresponde a un cuestionamiento directo de las concepciones ontológica y ética de la dimensión educativa a través de un proceso de autoconocimiento y autoevaluación de las prácticas educativas que han formado a docentes y 
estudiantes, al igual que a las que aplica en la escuela o colegio en el que desarrolla sus funciones.

Ser un docente y estudiante del sumak kawsay, o intentar generarlo, no es sencillo, puesto que conlleva la asunción voluntaria e intencional de un proceso político de transformación que, en el caso del contexto educativo ecuatoriano, por ejemplo, corresponde a ofrecer una formación que pretende que los estudiantes que egresan del sistema se caractericen por ser justos, innovadores y solidarios (ECUADOR, 2016). Tales particularidades generan una concepción diferente de las nociones de habilidades, destrezas, capacidades y competencias, que incentivan una relectura del proceso educativo, sus fines y puesta en práctica.

Ser justo, innovador y solidario como resolución de un proceso de formación es un propósito que se deriva de la propuesta de reforma educativa en el Ecuador, dirigida a transformar el sistema educativo en correspondencia con la realidad social del país plurinacional, caracterizado por un contexto intercultural en el que se ha dado lugar a la diversidad epistémica como base para la reconceptualización del sistema educativo. El propósito se relaciona con la transformación del habitante del Ecuador en un "sujeto político revolucionario" (Plan Nacional del Buen Vivir, 2013-2017), a través de la puesta en práctica de una economía social del conocimiento, que se dirige a la reapropiación social del conocimiento en un contexto intercultural crítico.

Las particularidades del proceso de implementación han requerido que el Ecuador genere una perspectiva geopolítica de la educación que haga posibles sus propósitos, encontrando resistencias de carácter ideológico que se fundamentan en el desconocimiento de la diversidad epistémica, provocando lo que se podría caracterizar como una clausura del imaginario social sobre la educación que se atribuye a la negación e invisibilización de la existencia de pensamiento a los indígenas, en la medida en la que aparentemente no hay una sistematización de las formas de conocimiento que han concebido, pero que también se extiende entre los habitantes de regiones diferentes caracterizadas por la migración y el mestizaje.

Esta resistencia ideológica signada por el racismo ha encontrado a la vez formas de confrontación a través de la ampliación de la noción de formación, propuesta a través de la inclusión de los siguientes ejes transversales en el currículo: a) la interculturalidad, b) formación de una ciudadanía democrática, c) la protección del medio ambiente, d) el cuidado de la salud y los hábitos de recreación de los estudiantes, e) la educación sexual en los jóvenes, proponiendo una atención 
especial a los ejes de igualdad y ambiente, comprendidos como sustentos de los otros (HERDOIZA, 2015).

Los ejes transversales se proponen con la intención de ofrecer una perspectiva sobre el contexto intercultural en el que se desarrollan las relaciones que componen el tejido social de un país que se concibe como plurinacional y que, en el caso de la educación, exige a los docentes el desarrollo de las asignaturas en correspondencia con los ejes mencionados.

La exigencia de aplicación de los ejes transversales provoca una serie de problemas en el momento de diseñar la malla curricular de las asignaturas que pretende ofrecer una institución, porque en el caso del contexto ecuatoriano, se conocen solo algunos aspectos del Plan Nacional del Buen Vivir, por lo que se minimiza la trascendencia para el proceso de transformación que dirige la política educativa, dificultando procesos como los de la adaptación curricular y la planificación de las clases.

En este sentido, el problema de la clausura del imaginario social sobre la educación toca las instancias que constituyen los cuerpos docentes de las instituciones, afectando directamente la formación de los estudiantes, pues al no tener docentes empoderados y comprometidos que pongan en práctica la reapropiación social del conocimiento, la promoción de seres justos, innovadores y solidarios que conformen una ciudadanía desde las concepciones del sumak kawsay, se dificulta.

Ante esta dificultad estructural, el Ministerio de Educación del Ecuador propone la creación de las universidades emblemáticas, que se dedican a la transformación del sistema educativo con la intención de proponer soluciones a una problemática que atañe a la producción de conocimientos a nivel pedagógico, tecnológico, cultural, científico.

Las universidades emblemáticas se crearon en el Ecuador como una estrategia para la realización de la trasformación del sistema educativo, y se sitúan en cuatro puntos que descentralizan la educación superior: en la Sierra, la Universidad Yachay para la ciencia y tecnología; en la Amazonía, la Universidad Ikiam, para las ciencias de la tierra; en Guayaquil, en la Costa, la Universidad de las Artes; y en Azogues, la Universidad Nacional de Educación, para la formación de formadores y docentes investigadores. La ubicación de las universidades se propone con intención de ampliar el acceso de diferentes poblaciones a la educación superior. 


\title{
5 Exploración crítica de su (posible) relación con un modelo que intenta humanizar el capitalismo
}

La relación del sumak kawsay con el concepto de reciprocidad genera una economía política diferente que se relaciona con la donación (MADROÑERO-MORILLO, 2015). Dar conlleva, en el contexto del pensamiento amerindio, una forma de práctica del saber que rebasa el intercambio simbólico, exponiendo una economía política del exceso, visible en la forma en que se establecen las relaciones de poder en una comunidad.

El poder entre algunas comunidades de América se caracteriza por el servicio más que por el mandato (CLASTRES, 1981); de esta manera, el líder de una comunidad se reconoce por su generosidad y capacidad de resolución de problemas, más que por el ejercicio de una autoridad soberana incuestionable; en este sentido, Clastres (1981) dice:

\begin{abstract}
¿Por qué las sociedades primitivas son sociedades sin Estado? Como sociedades completas, acabadas, adultas y no ya como embriones infra-políticos, las sociedades primitivas carecen de Estado porque se niegan a ello, porque rechazan la división del cuerpo social en dominadores y dominados. La política de los Salvajes se opone constantemente a la aparición de un órgano de poder separado, impide el encuentro siempre fatal entre la institución de la jefatura y el ejercicio del poder. En la sociedad primitiva no hay órgano del poder separado, porque el poder no está separado de la sociedad, porque es ella quien lo detenta como totalidad, con vistas a mantener su ser indiviso, de conjurar la aparición en su seno de la desigualdad entre señores y sujetos, entre el jefe y la tribu (p. 115).
\end{abstract}

La conjuración de la desigualdad representa una comprensión heterárquica del poder que se ejerce a través de la reciprocidad. El poder se propone en diáspora, sin un centro fijo que le permita establecerse, por lo que su movimiento es excéntrico e integra sin apropiar y homogeneizar; locomoción que provoca una economía de las relaciones diferente que no tiende a la acumulación sino a la inflexión y que hace de la inestabilidad una capacidad de transformación.

Una economía política de la inestabilidad hace de la pérdida un plus por el que la donación se manifiesta, pues el don excede la propiedad y la noción de valor de la concepción tradicional que la relaciona con el capital, considerado fundamento único para la generación de relaciones que sustenten un intercambio, 
lo que problematiza el ejercicio del poder cimentado en la estructura de la dominación que refleja el sistema dialéctico del "deber, la deuda y la obediencia" (AGAMBEN, 2008), que promueve la desigualdad y la institucionalización de la jerarquía.

El ecosistema de la política de transformación que promueve el sumak kawsay ofrece una alternativa al régimen de consumo sustentado en el deseo de poder. Como recalca Clastres (1981), esto implica: "Rechazo de la desigualdad, rechazo del poder separado" [...] Propuesto como "una preocupación constante en todas las sociedades primitivas [...] (pues) saben muy bien que, si renuncian a esta lucha, si cesan de contener esas fuerzas subterráneas que se llaman deseo de poder y deseo de sumisión, y sin cuya liberación no se puede comprender la irrupción de la dominación y la servidumbre, perderían su libertad" (CLASTRES, 1981, p. 115).

El "deber, la deuda, la obediencia", sustentan la economía de la dominación y su capitalización progresiva, estructura ante la que se propone incitar una prepulsión de emancipación que conlleva la acción política de un empoderamiento que se compone por la relación entre el ser justo, innovador y solidario, en el caso de la aplicación de esta intencionalidad de transformación.

Humanizar el capitalismo correspondería entonces, a provocar una remoción de la estructura de la dominación, que conllevaría pensar más allá de lo post colonial o lo descolonial, en la medida en que no se trata de la racionalización de la crisis, sino de la búsqueda o de la creación de propuestas de reafirmación de la vida como un valor que excede la resolución del capital, lo que conlleva pensar una concepción de humanidad alter-nativa y en el caso de la academia, unas ciencias humanas distintas, pues como subraya Betasamosake-Simpson (2014), desde el contexto del pensamiento indígena canadiense:

Si a la academia no sólo le preocupa proteger y mantener el pensamiento indígena, sino revitalizarlo en términos indígenas, como una forma de restitución por su papel histórico y contemporáneo como fuerza colonizadora (de la cual no veo evidencia), entonces la academia debe tomar una decisión consciente por convertirse en una fuerza descolonizadora en la vida intelectual de los pueblos indígenas, tomando parte en el desmantelamiento del colonialismo y protegiendo activamente la fuente de nuestro conocimiento - la tierra indígena (p. 22). 
En esta perspectiva, la reafirmación de la relación con ecosistemas de pensamiento que inciten la transformación de la realidad singular y comunal conlleva asumir la diversidad epistémica no solo como una forma de expresión de un multiculturalismo regulado, sino como la forma en la que sea posible construir geopolíticas del conocimiento dedicadas a la creación de otras ciencias, capaces de gestar relaciones alter-nativas con los saberes. En este sentido:

La 'Teoría' no es sólo una búsqueda intelectual - se teje en cada acción, movimiento y gesto, la presencia espiritual y la emoción, es contextual y relacional. Es algo íntimo y personal, está con los seres, en sí mismos, cuando asumen las responsabilidades que tienen para encontrar y generar significado y sentido en sus propias vidas (BETASAMOSAKE-SIMPSON, 2014, p. 7).

Dar sentido a la propia vida implica poner en práctica el don de la libertad, que se reafirma a partir de conceptos como los del sumak kawsay, cuya comprensión conlleva la asunción de una responsabilidad diferente pues no debe responder a un mandato sustentado en el deseo de poder o en la acumulación, ya que como destaca Betasamosake (2014), en "La Tierra como pedagogía":

La tierra, aki, es contexto y proceso. El proceso que se ofrece al alumno para que se dirija al conocimiento es profundamente espiritual en su naturaleza. Llegar a conocer conlleva la búsqueda de una comprensión de cuerpo presente que se practica en un contexto de libertad, cuando se realiza colectivamente, gesta generaciones de individuos amorosos, creativos, innovadores, autodeterminados, interdependientes y auto-reguladores. Crea comunidades de individuos con la capacidad de sostener y promover nuestras tradiciones políticas y sus sistemas de gobierno (BETASAMOSAKE-SIMPSON, 2014, p. 7).

La creación y gesta de comunidades de seres capaces de sostener tradiciones políticas alter-nativas y sistemas de gobierno desde una perspectiva ecosistémica compleja por la asunción de la diversidad epistémica, implica la comprensión de principios como los del sumak kawsay, Twaheed, Ubundu, desde un perspectivismo crítico que conlleva la práctica del pensamiento creativo como manifestación de la ética de la emancipación de la política de la libertad de una vida plena que se expresa en el buen vivir, no como finalidad, sino como intencionalidad transformativa continua de un ecosistema de pensamiento complejo, como refleja la Figura 6. 
Figura 6 - Ecosistema de pensamiento

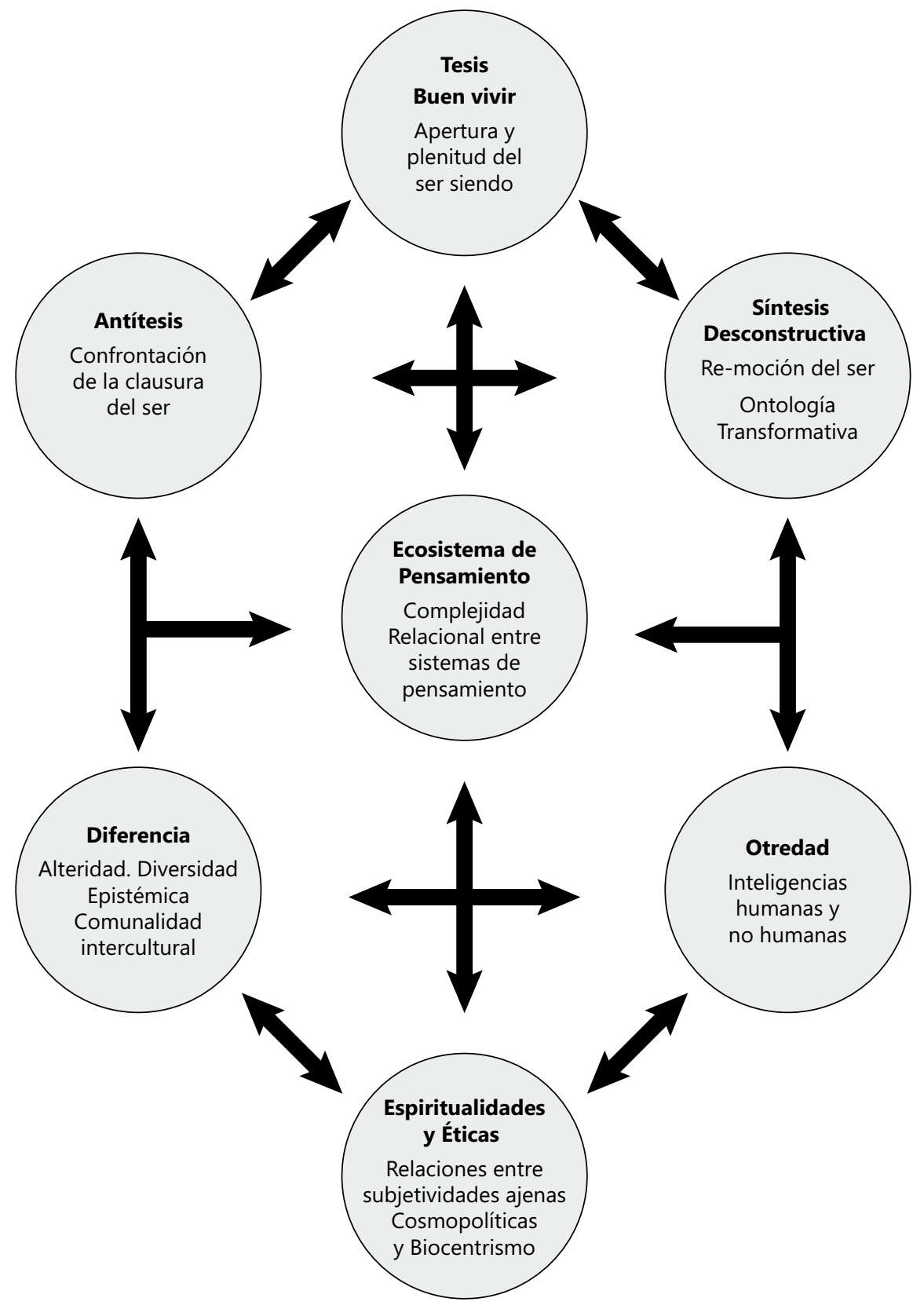

Fuente: Elaboración propia (2018) 


\section{Conclusiones}

La trascendencia del sumak kawsay para la política educativa reside en el hecho de que esta concepción puede ser considerada como una propuesta para pensar maneras alternativas para concebir la educación, entendida como un proceso de formación continuo.

El sumak kawsay expone voces de disenso ante los discursos dominantes sobre la educación, que lo relacionan con el perspectivismo, pues genera una concepción plural y diferente de la realidad educativa al relacionarla con una diversidad epistémica y que, en un proceso de formación, tiene que ver con cómo un docente o estudiante crean una relación con el conocimiento en contextos interculturales y con diversidades epistémicas y en cómo entiende y diseña una profesión y construye los sentidos de su vida.

Sumak kawsay es un concepto polilógico y dialógico, trama redes de interpretaciones sobre los sentidos de la vida y la relación con los demás, la naturaleza, la sociedad, la comunidad. Implica una confluencia de las formas de la razón y las inteligencias de la alteridad entre subjetividades ajenas, sean estas humanas o no humanas. Expone una constelación de significados y sentidos dedicados a recrear o inventar un sentido biocéntrico y plural de la vida con una intencionalidad impulsada por una cosmo-política que a su vez se sustenta en una posición política cosmo-referencial.

Una cosmo-política biocéntrica configura un modo particular de ser que expone una ética y una estética en tanto prácticas de un pensamiento alter-nativo, pues son prácticas de transformación ontológica del ser que se revelan en el concepto kay que expresa la relación intensiva con un tiempo presente que expone una forma de comprensión con la tierra que se expresa en el concepto de Pacha Mama, asumido como un tiempo para la creación y el cuidado de sí mismo y de los demás, desde una reciprocidad y disidencia prácticas que se expresan en el don.

La reciprocidad como disidencia práctica es una característica del pensamiento amerindio, relacionada con "procesos de alteración diferencial" (VIVEIROS DE CASTRO, 2002), que conllevan asumir una alteridad política; en este sentido, la relación política es un conflicto permanente sin necesidad de control o sumisión, debido a que el poder no es un destino o una finalidad, por lo que no depende de conservar una estructura para la conservación de la soberanía.

El conflicto es un antagonismo afirmativo porque se sustenta en la búsqueda de un don. El don establece relaciones que rebasan la economía acumulativa. En este 
sentido, el don es una expresión de generosidad que no implica el ejercicio de un poder superior. La generosidad provoca una práctica heterárquica y un tiempo heurístico que hace posible una transformación de la realidad singular o en la comunidad; de ahí que sea un poder sin soberanía.

Este concepto de poder implica un concepto diferente de ley, justicia, derecho, deber y libertad. En este sentido, es una comprensión alter- nativa sobre la responsabilidad que, en el contexto educativo, hace que las relaciones entre docentes y estudiantes cambien, porque el don implica una práctica formativa que involucra una relación creadora, - de generosidad - con el conocimiento. No hay un propietario del sentido de la verdad, ni una verdad única, porque la relación con la verdad se sustenta en una diversidad epistémica práctica.

La relación con una diversidad epistémica es una práctica política para la transformación del conocimiento, provoca una educación transformativa y genera un ecosistema de formación y creación de conocimientos. En este sentido, compone un ecosistema educativo transformativo que se representa con el concepto Pacha Mama, comprendido como el tiempo para la creación y el cuidado de sí y los otros.

Pacha Mama y Sacha Mama, la selva, la tierra, son madre-pensamiento, - madre del proceso de formación-, pues como Betasamosake-Simpson (2014) dice, se trata de "La tierra como Pedagogía". Concepción que nos recuerda el pensamiento de Juan Chiles, un chamán indígena - líder ancestral de la comunidad de los Pastos entre la frontera entre Colombia y Ecuador -, cuando dice sobre la formación de la comunidad desde una ontología transformativa que "Somos como el agua, somos como la piedra, somos como la espuma, pero somos el río", sustentando la concepción en donde "el contexto es el currículo" (BETASAMOSAKE-SIMPSON, 2014, p. 10).

Sumak kawsay es un concepto trascendental para la educación porque expone un pensamiento dedicado a la invención o creación de sentidos sobre la vida; su relación con una política educativa se sustenta en un pensamiento ético y estético que implica la praxis de una ontología transformativa que incentiva una toma de decisión por vivir de otro modo y sustenta la decisión política de aprender a vivir de nuevo, generando transformación en docentes y estudiantes que asumen la dificultad de la desconstrucción de un sistema educativo y su representación de la realidad.

Sumak kawsay y deconstrucción están relacionados por una remoción del ser que provoca cambios en la configuración del sistema epistémico de la razón, que 
re-mueve los conceptos en dimensiones ontológicas, éticas y políticas, por lo que la subjetividad se recrea por la experiencia de un aprendizaje pre-ontológico de los sentidos sobre la vida que se da por la relación voluntaria con un pensamiento ancestral, asumido como otra temporalidad de formación.

La sabiduría ancestral de las comunidades habla de esta experiencia en los mitos y sistemas complejos de su diversidad epistémica e implica posibilidades para la concepción de una pedagogía transformativa expuesta en conceptos como sumak kawsay, un hilo en la trama de la constelación de sentidos sobre la vida. 


\title{
A transcendência de sumak kawsay para a nossa compreensão da educação, ética e política
}

\section{Resumo}

"Sumak kawsay" é uma concepção que ao longo dos últimos anos gerou considerável interesse em contextos políticos, econômicos, educacionais e de pesquisa, recebendo atenção de ativistas e acadêmicos devido à "ruptura" que causa na concepção atual da ordem mundial. Em diferentes contextos, assume-se como algo que deve ser alcançado através de um processo de transformação sobre o modo como as representações sociais são construídas a nível singular, comunitário e planetário, propondo "pensar de outra maneira" sobre os conceitos de modernidade que tomamos como certo, e promovendo um chamado para a restauração ou invenção do sentido da experiência, cultura, vida, a harmonia possa existir, bem como o respeito pela natureza.

Palavras-chave: Sumak kawsay. Ética. Política. Educação. Transformação.

\section{The implications of sumak kawsay for our understanding of education, ethics, and politics}

\begin{abstract}
"Sumak kawsay" is a conception that in the course of recent years has generated considerable interest in political, economic, and educational contexts, as well as an increasing attention on the part of activists and academics due to the "rupture" it causes on the world order. In different contexts, it is assumed as something that must be achieved through a process of transformation about the way in which social representations are constructed at a singular, community and planetary level by proposing to "think otherwise" the concepts of modernity that we take for granted, and by promoting a call to the restoration or invention of the sense of experience, culture, and life, in such a way that harmony and respect for nature become viable.
\end{abstract}

Keywords: Sumak kawsay. Ethics. Politics. Education. Transformation. 


\section{Referencias}

ACOSTA, A. El Buen Vivir, una oportunidad para construir. Ecuador Debate, Quito, n. 75, p. 33-47, 2008.

AGAMBEN, G. El reino y la gloria: por una genealogía teológica de la economía y del gobierno. Valencia: Pre-textos, 2008.

BETASAMOSAKE-SIMPSON, L. Land as pedagogy: Nishnaabeg intelligence and rebellious transformation. Decolonization: Indigenity, Education and Society, [s. l.], v. 3, n. 3, p. 1-25, 2014.

CLASTRES, P. Investigaciones en antropología política. Barcelona: Gedisa, 1981.

CORTEZ, D. La construcción social del Buen Vivir (Sumak Kawsay) em Ecuador: genealogía del diseño y gestión política de la vida. Plataforma del Buen Vivir. 2011. Disponible en: http://www.plataformabuenvivir.com/ wpcontent/uploads/2012/07/CortezGenealogiaBuenVivir11.pdf. Acceso en: 25 abril 2018.

DERRIDA, J. Fuerza de ley. El "fundamento místico de la autoridad". Madrid: Tecnos, 2008.

ECUADOR. Ministerio de Educación Nacional. Currículo de los niveles de educación obligatoria. Quito, 2016.

GROSFOGUEL, R. Del extractivismo económico al "extractivismo epistémico" y "extractivismo ontológico". Tabula Rasa, Bogotá, v. 1, n. 4, p. 33-45, 2016. https://doi.org/10.15304/ricd.1.4.3295

GUDYNAS, E. Buen Vivir: germinando alternativas al desarrollo. La Paz: ALAI, 2011.

HERDOIZA, M. Construyendo igualdad en la educación superior: fundamentación y lineamientos para transversalizar los ejes de igualdad y ambiente. Quito: Senescyt : Unesco, 2015.

KONTOPOULOS, K. The logics of social structure. London: Routeledge, 1993. 
LAJO, J. Un modelo sumaq kawsay de gobierno. Socios, 29 ago. 2011. Disponible en: http://www.voltairenet.org/article171245.html. Acceso en: 8 maio 2018.

LIU-SHING, I. El principio de reciprocidad y la globalización d el derecho desde la perspectiva de la regla de oro. Persona y Derecho, [s. l.] v. 60, p. 1927, 2009.

MACAS, L. Sumak kawsay: recuperar el sentido de vida. In: El Buen Vivir de los pueblos indígenas andinos. Plataforma del Buen Vivir. 2010. Disponible en: http://www.plataformabuenvivir.com/wpcontent/uploads/2012/07/ MacasSumakKawsay2010.pdf. Acceso en: 10 maio 2018.

MADROÑERO-MORILLO, M. Flujos de ingobernabilidad: don y derecho alter-nativo. FLACSO, Quito, v. 2, n. 2, p. 71-92, 2015.

RIVERA-CUSICANQUI, S. Ch'ixinakax utxiwa: una reflexión sobre prácticas y discursos descolonizadores. Buenos Aires: Tinta Limón y Retazos, 2010.

RUANO COLLADO, J.; MORILLO MADROÑERO, M.; GONZÁLES ÁLVAREZ, F. Educación transdisciplinar: formando en competências para el buen vivir. Ensaio: Avaliação e Políticas Públicas em Educação, Río de Janeiro, v. 26, n. 100, p. 619-44, jul./set. 2018. https://doi.org/10.1590/s010440362018002601487

STIEGLER, B. Taking care of youth and the generations. Palo Alto: Stanford University Press, 2010.

TROUILLOT-ROLPH, M. Transformaciones globales: la antropología y el mundo moderno. Popayán: Universidad del Cauca, 2011.

VIVEIROS-DE-CASTRO, E. A inconstancia da alma selvagem e outros ensaios de antropología. São Paulo: Cosac Naify, 2002. 


\section{Informações dos autores}

Mario Marino Madroñero Morillo: Doctor en antropología por la Universidad del Cauca en Colombia. Docente de la Universidad Nacional de Educación del Ecuador. Contato:mario.madronero@unae.edu.ec

iD https://orcid.org/0000-0001-5828-688X

Enma Polonia Campozano Aviles: PhD student KU Leuven, Laboratory for educational and society of Faculty of Psychology and Educational Sciences, Belgium. Docente de la Universidad Nacional de Educación del Ecuador. Contato: enma.campozano@unae.edu.ec

iD https://orcid.org/0000-0003-0233-8585

Julio Uyaguari: Estudiante de la Licenciatura en Educación General Básica de la Universidad Nacional de Educación del Ecuador. Contato: jruyaguari@unae.edu.ec

iD https://orcid.org/0000-0001-8478-9501 